Pain relief motion and function after rotator cuff repair or reconstruction may not persist after 16 years

Borgmastars $N$, et al. CORR (2010)10, 2678-2689

Short to medium term rotator cuff repair is said to relieve pain in $82 \%$ to $97 \%$ of patients and provides normal or near normal function in $82 \%$ to $92 \%$. In order to address the unknown long term results of function and pain relief 75 patients ( 55 men, 20 women) who underwent rotator cuff repair between 1980 and 1989 were retrospectively reviewed. The mean age at surgery was 52 years and the minimum follow-up, 16 years. $37 \%$ had pain relief lasting for 20 years. In the remainder, pain relief lasted an average of 14 years. Initial flexion and abduction strength increased post-operatively but during long term follow-up decreased to less than preoperative levels. Thus, early high functional scores did not persist although pain relief was long standing.

Birmingham hip resurfacing: the prevalence of failure Carrothers $A D$, et al. JBJS (Bx)2010, 10, 1344-1350

141 surgeons and 5000 Birmingham hip resurfacings constituted the study group for analysis of modes, prevalence, gender differences and times to failure, requiring revision. 182 hips required revision (3.6\%). The most common cause for revision was a fracture of the neck of the femur $(1.1 \%)$ followed by loosening of the acetabular component $(0.6 \%)$, AVN-collapse of the femoral head $(0.6 \%)$, loosening of the femoral component $(0.4 \%)$ infection $(0.3 \%)$ pain with aseptic lymphocytic and associated lesions (AVAL) (0.3\%) and loosening of both components $(0.1 \%)$.

The prevalence of revision was significantly higher in women $(\mathrm{W}=5.7 \% ; \mathrm{M}=2.8 \%)$. Mean time to failure was 2.9 years with fracture of the femoral neck occurring earlier than all other causes. Failure in men occurred earlier (2.1 years) than women (3.6 years).

Prevalence comparison of accompanying lesions between primary and recurrent anterior dislocation in the shoulder Kim DS, et al.

Am J Sports Med (2010) 38, 2071-2076

The study groups comprised 33 in the primary dislocation and 111 in the recurrent dislocation group. All patients had preoperative MR arthrography and diagnostic arthroscopy. In the primary group there were 9 Bankhart lesions, 9 ALPSA, 4 bony Bankharts and 1 adhesive ALPSA lesion. In the recurrent dislocation group there were 68 Bankhart lesions, 11 free ALPSA lesions, 13 bony Bankharts and 16 adhesive ALPSA lesions and 1 glenoid articular rim fracture. The differences were statistically significant $(\mathrm{P}=0002)$ in the two groups as was the prevalence of Hill-Sachs lesions. $2 \mathrm{HAGL}$ lesions were found in the primary group and none in the recurrent dislocators. Although there was no statistical difference in SLAP lesions between the two groups more were encountered in the recurrent group;28 to 8 . Bony lesions were more common with the recurrent dislocations. MR arthrography showed high accuracy in both groups.

\section{Osteosarcoma in very young children}

Leo $K$, et al.

Cancer (2010) 116, 5316-5324

Osteosarcoma, the most common primary malignant tumor of bone in childhood and adolescence has a prevalence that significantly varies with age ranging from 2.4 cases per million per year from 5 to 9 years upward and increasing to 8.2 cases per million per year from 15 to 19 years. Under 5 years of age the prevalence is 0.4 cases per million per year. 27 children under five years with osteosarcoma were identified from a cohort of 2706 patients drawn from three European countries. The median age at diagnostic biopsy was 3.9 years. Nine presented with a pathologic fracture. 20 of the tumors were considered large involving more than one third of the bone, with femur, humerus and tibia representing the most frequently involved sites in descending order of frequency.

Of the six patients with telangiectatic osteosarcoma, four presented with a pathologic fracture. Although there was no correlation between patients' age, gender, duration of symptoms, size, location and presence or absence with pathologic fracture and outcome, those treated after 2000 had significantly improved outcomes. The authors compared their findings with 27 osteosarcoma preschoolers described in the existing literature. Taking into consideration patients with osteosarcoma in all age groups, large osteosarcomas comprised one third of the patient population, while in the preschoolers previously described and in the group presented here, $75 \%$ of the tumors were greater than or equal to one third of the bone involved. As with other age groups, a good histologic response to chemotherapy was a critical favorable prognostic factor.

Abstracted by: M. Sundaram, M.D. February 2011 